\title{
Influence of unbalance levels on nonlinear dynamics of a rotor-backup rolling bearing system
}

Fonseca, Cesar A. ; Santos, Ilmar; Weber, Hans I.

Published in:

Journal of Sound and Vibration

Link to article, DOI:

10.1016/j.jsv.2017.01.020

Publication date:

2017

Document Version

Peer reviewed version

Link back to DTU Orbit

\section{Citation (APA):}

Fonseca, C. A., Santos, I., \& Weber, H. I. (2017). Influence of unbalance levels on nonlinear dynamics of a rotorbackup rolling bearing system. Journal of Sound and Vibration, 394, 482-496.

https://doi.org/10.1016/j.jsv.2017.01.020

\section{General rights}

Copyright and moral rights for the publications made accessible in the public portal are retained by the authors and/or other copyright owners and it is a condition of accessing publications that users recognise and abide by the legal requirements associated with these rights.

- Users may download and print one copy of any publication from the public portal for the purpose of private study or research.

- You may not further distribute the material or use it for any profit-making activity or commercial gain

- You may freely distribute the URL identifying the publication in the public portal 


\title{
Influence of Unbalance Levels on Nonlinear Dynamics of a Rotor-Backup Rolling Bearing System
}

\author{
Cesar A. Fonseca*, Ilmar F. Santos** \\ Dept. of Mechanical Engineering, DTU, Niels Koeppel Allé, 404, 2800, Lyngby, Denmark \\ Hans I. Weber \\ Mech. Engineering Dept., PUC-Rio, Rua Marquês de São Vicente, 225, Rio de Janeiro, \\ RJ, Brazil
}

\begin{abstract}
Rotor drops in magnetic bearing and unbalance in rotors have been the subject of study for many years. The combination of these two well-known phenomena has led to an interesting chaotic response, when the rotor touches the inner race of the backup bearing. The present work explores the nonlinear rotor-backup-bearing dynamics both theoretically and experimentally using a fully instrumented test rig, where the position of the shaft, its angular velocity and the contact forces between the shaft and the backup bearing are sampled at $25 \mathrm{kHz}$. The test rig is built by a removable passive magnetic bearing, which allows for the simulation of a magnetic bearing failure (loss of carrying capacity and subsequent rotor fall). A theoretical approach is given beforehand and forms the basis of the study. The theoretical and numerical analyses are shown through Poincaré maps and double sided spectra. The latter are important to characterize the condition at different levels of unbalance between forward and backwards whirl. Our preliminary results indicate that for small levels of unbalance the rotor oscillates at the bottom of the backup bearing. When the levels of unbalance increase, the dynamical behaviour of the rotor changes; this leads to extremely harmful conditions, since the rotor can be lifted from the bottom of the bearing (contact state)
\end{abstract}

\footnotetext{
*Principal corresponding author

** Corresponding author

Email addresses: cefonse@mek.dtu.dk (Cesar A. Fonseca), ilsa@mek.dtu.dk (Ilmar F. Santos), hans@puc-rio.com (Hans I. Weber)
} 
and fall again, impacting on the inner race innumerable times without reaching a steady state. Finally the presented results are discussed from the point of view of nonlinear dynamics applied to the practical use.

Keywords: Safety bearings, impact, friction, contact mechanics, nonlinear dynamics, magnetic bearing.

\section{Introduction}

The possibility of using magnetic bearing rotors in industrial applications cannot be considered correctly without the use of proper backup bearings. Applications are various, such as in reaction wheels, in centrifuges, in energy efficiency machines, as cited for instance by Gasch [7] and Schweitzer and Maslen [23]. For all these machines, a safety element has to be installed to prevent failures such as power loss, which would cause the shaft to fall. The safety element consists normally of a rolling bearing with inner race diameter greater than the shaft diameter, but smaller than the one of the magnetic bearing. It is designed to withstand the loads and impacts of a rotor. It also protects the whole system and prevents even more disastrous situations.

Throughout the years, several works have been published on this subject. A good overview of the state of rotordynamics research of rub-related phenomena is given in Muszynska [19]. In the work of Johnson [15], a vertical shaft was studied analytically and he investigated the changes in the radial peaks when a bearing with clearance is introduced. Black [1] also considered a rotor with a clearance stator but added friction on the contact. He conducted an analytical study, whose results about synchronous and counter whirl were verified experimentally. Also in reference [29], a gyro pendulum with a piecewise linear model was investigated with good agreement with experimental results. Choy and Padovan [2] presented a numerical investigation of the interaction between the rotor and the casing of the bearing wall, in which different parameters where tested, such as imbalance and friction coefficients.

By employing a proper impact model, the occurrence of chaotic behavior was reported by Goldman [9]. Moreover Piccoli and Weber [20] successfully investigated the identification of chaotic motion with Lyapunov exponents and Poincaré diagrams experimentally. In Jiang and Ulbrich [14], the onset of dry friction whip was investigated with an unbalanced rotor to stator contact explaining the recurrence using the multiple scales method. Regarding rotor 
drops, the work from Pradetto [21] describes the resulting phenomena of the drop of a one-ton rotor as its backup bearing. Fumagalli [5] tested the performance of the ball bearing instead of a plain circular one as a backup bearing when the rotor drops, slides and tumbles on its inner race. His results led to the information about the rotor's whirl and motion as well as the contact forces at each impact. More recently, [24] presented a simulation with experimental validation of a 9-ton-active-magnetic-bearings-rotor drop then compared and validated the proprietary code to several drop test as a tool for future reliability tests. [13] analyses the rotor-to-stator impact of a turbo-machinery with a thermo coupled model with dynamical system. Their findings explores the nonlinear nature of the impact response and the interaction with the blades of the rotor with the casing.

In combination with Active Magnetic Bearings (AMB) technology as mentioned by Schweitzer [23] and in [22], it is clear that safety bearings are an important subject of study. Chapter 27 in the book of Gasch et al. [6] also treats the cases, in which the rotor starts impacting on the stator including resonance passage and sudden unbalance. Ishii and Kirk [12] analyzed numerically the transient response of a rotor drop. There it is cited that low damping causes backwards whirl that leads to high-magnitude forces. The work of [26] presents a very detailed mechanical model of a flywheel with two safety bearings with power loss. The authors conducted a series of simulations, whose response changed according to the parameters such as friction and the preload applied. Ginzinger [8] developed an active actuator to avoid rubbing and thus reduce the severity of the contact. He applies a feedback control with a powerful magnetic actuator to smoothen the transition from an impact-free to full contact eliminating impacts. Moreover, in Keogh [16], a comprehensive study of different performances of auxiliary bearings was presented. Non-conventional geometries of the backup bearing were considered by Simon [25], and later by Zülow [30] and then the new kind of bearing with pins is presented by Lahriri [17] and analyzes the forces that the structure receives on a sleeve backup bearing. The rotor is externally impacted and the dynamics of the first impacts and the high radial forces in that generated by the backwards whirl is assessed. In Fonseca et al. [4] it is shown that the same pins help to surpass the critical speed avoiding the backwards whirl. However, in most industrial operating machines, the usual safety bearing is a rolling bearing element, and the rotor will lay down at the bottom allowing it to rotate without much damage to the system. An analysis of the rigid rotor on a safety bearing showing chaotic behavior is presented in reference [28]. 
Later the contact between the shaft and the inner race was modeled using a nonlinear matrix equation and presented by Cole et al. [3], whose authors analyzed different configurations of rolling bearings in order to identify the dynamics conditions of the rotor during impacts events and to try to specify a proper backup bearing. More recently, Inayat-Hussain [11] gives an insight about the response of a two dimensional rotor to different imbalance levels with bifurcation diagrams, which show chaotic behavior when impacting on the wall of the backup bearing. In his thesis, van Rensburg [27] applied to his rotor model and AMB test rig several rotational speeds and showed different dynamical behaviors and harmful cases. In Hakwings et al. [10] the result of a series of drop tests of $140 \mathrm{~kW}$ flywheel on a backup bearing is presented and illustrate the dynamical behavior of the rotor in the case of total failure and unassisted spin down.

In the present work, a theoretical and an experimental study of a passive magnetic bearing rotor colliding on the ball bearing inner race is presented. The failure of the rotor will be induced by the rapid removal of the passive magnetic bearing, letting the rotor fall on the backup bearing. The tests will be compared with simulated results coming from a model of the rotor as a rigid body impacting on an compliant surface of a compliant housing support. The main contribution of this work is to show that different levels of unbalance change the dynamical behavior of the system at least into three separate regions, and some of them may lead to harmful situations. In order to create these different levels of unbalance, small masses are added to the disk of the rotor, but the velocity was kept constant. The findings are best portrayed in bifurcation diagrams that describe the changes. Also, these features appear on orbit plots, doubled-sided spectra and time-varying force plots.

In Section 2, the dynamical model for the rotor-backup-bearing interaction is developed. In Section 3, the simulation and experimental results are shown and discussed. Finally, in Section 4, we present our conclusions.

\section{Modeling the rotor-housing kinematics}

\subsection{The shaft}

In Figures 1a and b, a schematic description of the rotor is made to reproduce the assembled test rig. The rotor is supported by one spherical ball bearing at point $O$ and one movable passive magnetic bearing at point

C. The backup bearing is mounted between them at point B. The rotor is 


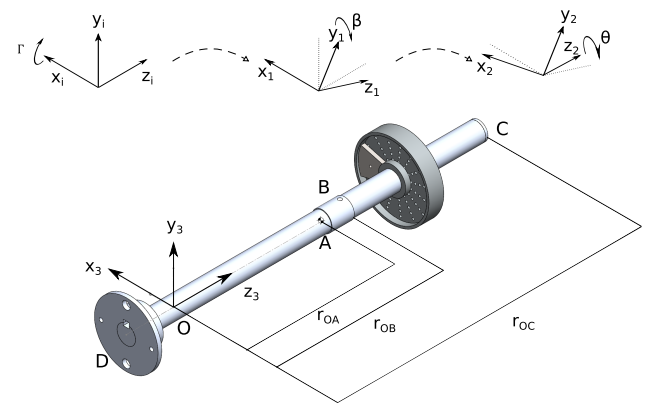

a)

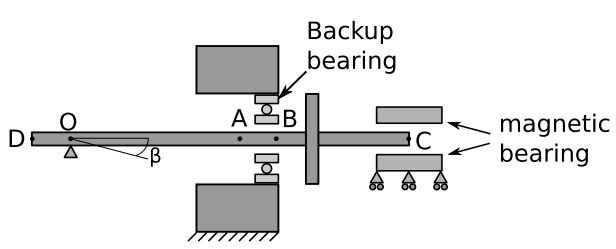

b)

Figure 1: The moving frame references and the points of interests, $A, B$ and $C$, where forces are present.

modeled as a rigid body and external forces from the magnets, imbalance and coupling are applied at points $\mathrm{C}, \mathrm{A}$ and $\mathrm{D}$ respectively. In these figures the reference frames used are shown and the moving reference frame $B 3$ fixed to the rotating shaft and positioned at the supporting point of the rotor. Figure 2a is a photograph of the complete test rig and Figure $2 \mathrm{~b}$ is another one taken above the magnetic bearing and pointed to the backup bearing.

The rotor is only allowed to rotate according to the following three angular degrees of freedom: $\Gamma(t), \beta(t), \theta(t)$, around the respective coordinate axes $x_{i}, y_{1}, z_{2}$, for which we define the angular velocities

$$
{ }_{I} \dot{\boldsymbol{\Gamma}}=\left[\begin{array}{lll}
\dot{\Gamma}(t) & 0 & 0
\end{array}\right]^{T}, \quad{ }_{B 1} \dot{\boldsymbol{\beta}}=\left[\begin{array}{lll}
0 & \dot{\beta}(t) & 0
\end{array}\right]^{T} \text { and } \quad{ }_{B 2} \dot{\boldsymbol{\theta}}=\left[\begin{array}{lll}
0 & 0 & \dot{\theta}(t)
\end{array}\right]^{T} .
$$

Then the rotational matrices ${ }_{I} T_{\Gamma},{ }_{B 1} T_{\beta}$ and ${ }_{B 2} T_{\theta}$ are defined as

$$
\begin{gathered}
{ }_{I} \mathbf{T}_{\Gamma}=\left[\begin{array}{ccc}
1 & 0 & 0 \\
0 & \cos \Gamma(t) & \sin \Gamma(t) \\
0 & -\sin \Gamma(t) & \cos \Gamma(t)
\end{array}\right], \quad{ }_{B 1} \mathbf{T}_{\beta}=\left[\begin{array}{ccc}
\cos \beta(t) & 0 & -\sin \beta(t) \\
0 & 1 & 0 \\
\sin \beta(t) & 0 & \cos \beta(t)
\end{array}\right], \\
{ }_{B 2} \mathbf{T}_{\theta}=\left[\begin{array}{ccc}
\cos \theta(t) & \sin \theta(t) & 0 \\
-\sin \theta(t) & \cos \theta(t) & 0 \\
0 & 0 & 1
\end{array}\right] .
\end{gathered}
$$

Each external force will generate a moment with respect to $O$ and the relevant position vectors are given by 


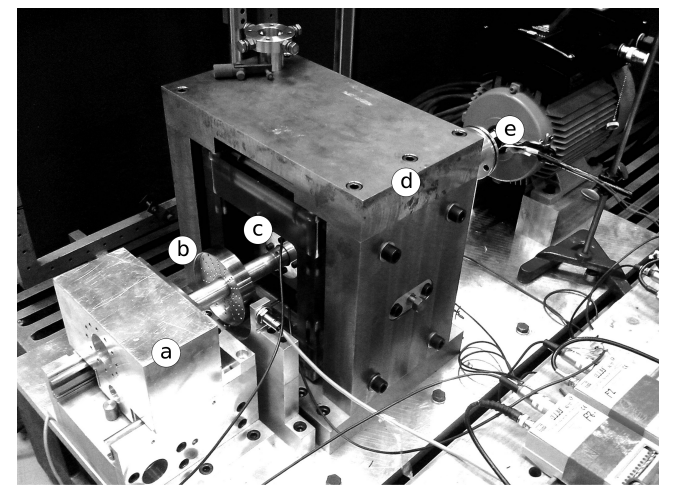

a)

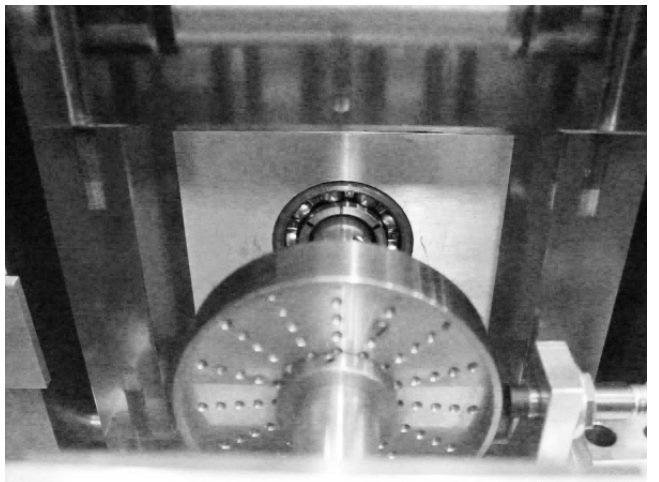

b)

Figure 2: a) Photograph from the test rig assembled for tests with different backup bearings. a - Passive magnetic bearing; b - Rotor with disk; c - Backup bearing. Here the backup bearing is a plain wall bearing; $\mathrm{d}$ - Backup bearing housing; e - AC Motor. b) Details from the shaft and the rolling bearing backup bearing.

$$
\begin{gathered}
{ }_{B 3} \mathbf{r}_{O A}=\left[\begin{array}{lll}
r_{O A, x} & 0 & r_{O A, z}
\end{array}\right]^{T}, \quad{ }_{B 3} \mathbf{r}_{O B}=\left[\begin{array}{lll}
r_{O B, x} & r_{O B, y} & r_{O B, z}
\end{array}\right]^{T} \text { and } \\
{ }_{B 3} \mathbf{r}_{O C}=\left[\begin{array}{lll}
0 & 0 & r_{O C, z}
\end{array}\right]^{T} .
\end{gathered}
$$

The inertia tensor referred to the supporting point is

$$
{ }_{B 3} \mathbf{I}_{O}=\left[\begin{array}{ccc}
I_{x x} & 0 & -I_{x z} \\
0 & I_{y y} & 0 \\
-I_{z x} & 0 & I_{z z}
\end{array}\right]
$$

where $I_{x z}=m_{u} r_{u} l_{O D}$. The absolute angular velocity represented in the coordinates of the moving reference frame B3 (where the inertia tensor is constant) is given by

$$
\boldsymbol{\omega}_{B 3}={ }_{B 3} \dot{\boldsymbol{\Gamma}}+{ }_{B 3} \dot{\boldsymbol{\beta}}+{ }_{B 3} \dot{\boldsymbol{\theta}}=\left[\begin{array}{c}
\cos (\theta) \cos (\beta) \dot{\Gamma}+\sin (\theta) \dot{\beta} \\
-\sin (\theta) \cos (\beta) \dot{\Gamma}+\cos (\theta) \dot{\beta} \\
\sin (\beta) \dot{\Gamma}+\dot{\theta}
\end{array}\right] .
$$

The absolute acceleration written in B3 is written as:

$\dot{\boldsymbol{\omega}}_{B 3}=\left[\begin{array}{c}-\dot{\theta} \sin (\theta) \cos (\beta) \dot{\Gamma}-\cos (\theta) \dot{\beta} \sin (\beta) \dot{\Gamma}+\cos (\theta) \cos (\beta) \ddot{\Gamma}+\dot{\theta} \cos (\theta) \dot{\beta}+\sin (\theta) \ddot{\beta} \\ -\dot{\theta} \cos (\theta) \cos (\beta) \dot{\Gamma}+\sin (\theta) \dot{\beta} \sin (\beta) \dot{\Gamma}-\sin (\theta) \cos (\beta) \ddot{\Gamma}-\dot{\theta} \sin (\theta) \dot{\beta}+\cos (\theta) \ddot{\beta} \\ \dot{\beta} \cos (\beta) \dot{\Gamma}+\sin (\beta) \ddot{\Gamma}+\ddot{\theta}\end{array}\right]$. 


\subsection{The inner and outer housing}

In order to determine and measure the force between the rotor shaft and the inner race, the backup bearing is mounted on a special casing, where there are four force transducers. It is inside a block that is mounted inside a frame, called an outer house. Then, the inner house is only allowed to move vertically inside it. Subsequently, the outer house moves only horizontally. Figures $3 \mathrm{a}$ and $\mathrm{b}$ show a scheme of the complete assembly.

The elastic elements $k_{f t}$ represent the force transducers positioned between the bodies; the damping elements $c_{h}$ and $c_{v}$ represent the structural damping. These damping terms are present due to the four beams that make the inner house slide vertically and the outer house horizontally, two terms for each direction.

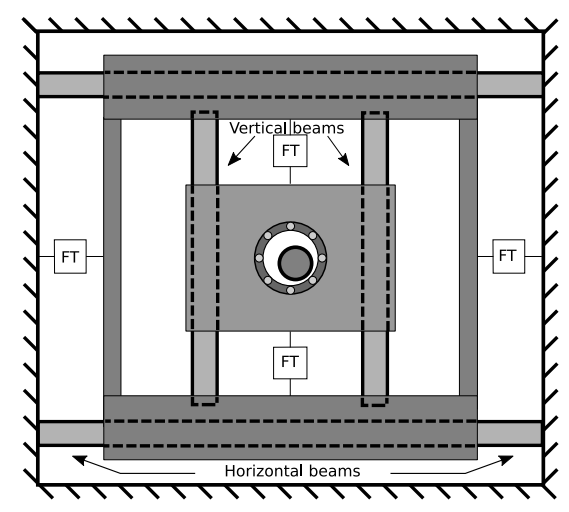

a)

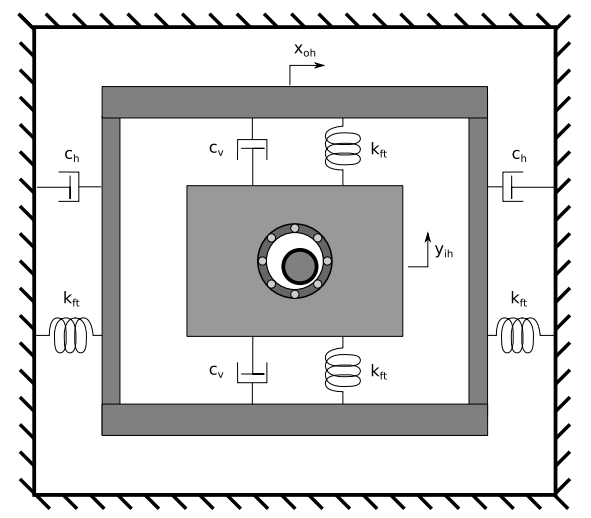

b)

Figure 3: Assembly of the inner housing and the outer housing.

The dynamics of both housings is included in the mechanical model. The dynamical coupling between the inner house and the rotor is introduced by the impact forces, the normal force $N$ and the friction force $F_{\text {fric }}$. The beams that support the housings have the stiffness calculated as clamped-clampled beam $K_{\text {beam }}=\frac{48 E I}{F a^{2}(3 l-4 a)}$, and the damping coefficients are approximated by:

$$
c_{h}=2 \zeta \sqrt{K_{\text {beam }}\left(m_{i h}+m_{o h}\right)} \text { and } c_{v}=2 \zeta \sqrt{K_{\text {beam }}\left(m_{i h}\right)} .
$$

Thus the equations of motion for both housings are written as follows

$$
\begin{aligned}
m_{i h} \ddot{y}_{i h} & =-2 k_{f t} y_{i h}-2 c_{v} y_{i h}-m_{i h} g+N \sin \alpha+F_{\text {fric }} \cos \alpha . \\
\left(M_{o h}+m_{i h}\right) \ddot{x}_{o h} & =-2 k_{f t} x_{o h}-2 c_{h} x_{o h}+N \cos \alpha-F_{\text {fric }} \sin \alpha .
\end{aligned}
$$




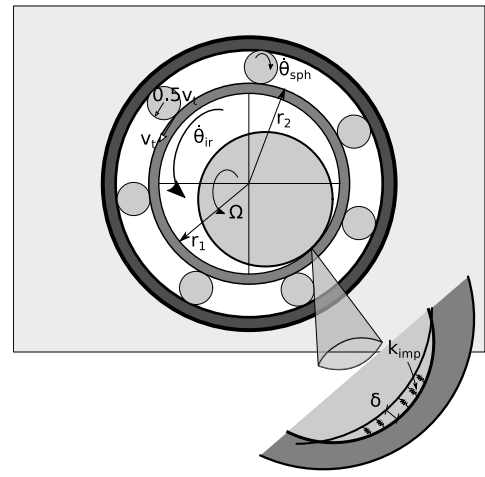

a)

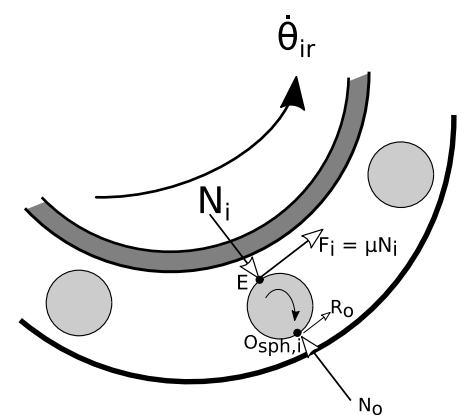

b)

Figure 4: a) The shaft inside the inner housing in contact with the inner race. b) The forces acting at each ball of the backup bearing.

Consequently, the radial term $r_{r}$ can be calculated and allows one to know whether the system is impacting or not, and it is equal to

$$
r_{r}=\sqrt{\left.\left(\beta l_{O B}-x_{o h}\right)^{2}+\left(-\Gamma l_{O B}\right)-y_{i h}\right)^{2}} .
$$

The impact is analyzed at each time step if the rotor displacement at the position of the backup bearing is greater or equal to the radial gap, $\delta \geq r_{0}=r_{1}-r_{r}$. The impact is modeled following a stepwise elastic model proposed by [18], that is:

$$
F_{\text {imp }}=N=k_{\text {imp }} \delta^{3 / 2}\left(1+\frac{3\left(1-e^{2}\right)}{4} \frac{\dot{\delta}}{\dot{\delta}^{-}}\right), \quad \text { if } \quad \delta \geq 0,
$$

in which the stiffness coefficient is given as:

$$
k_{i m p}=\frac{4}{3\left(\frac{1-\nu_{s}^{2}}{E_{s}}+\frac{1-\nu_{i}^{2}}{E_{i}}\right)}\left(\frac{r_{s} r_{1}}{r_{1}-r_{s}}\right)^{1 / 2} .
$$

and $e$ is the coefficient of restituition. The model of impact has the advantage of avoiding the determination of a damping factor and relies only on the value of the coefficient of restitiution, which can be determined easier through experiments. On other side, it is a piece-wise model that can lead to numerical instabilities in the numerical integration in time. Therefore it is 
recommended to employ techniques like Filipov or manipulate the tolerances into the computational code.

Therefore, the forces acting on the mechanical model are the rotor's own weight, the magnetic force and the damping force plus the impact forces

$$
\begin{gathered}
{ }_{I} \mathbf{F}_{g}=\left[\begin{array}{c}
0 \\
-m g \\
0
\end{array}\right],{ }_{I} \mathbf{F}_{m a g}=\left[\begin{array}{c}
-K \cos \alpha \\
-K \sin \alpha \\
0
\end{array}\right],{ }_{I} \mathbf{F}_{\text {damp }}=\left[\begin{array}{c}
-c \cos \rho \\
-c \sin \rho \\
0
\end{array}\right], \\
{ }_{I} \mathbf{F}_{\mathrm{imp}}=\left[\begin{array}{c}
N \cos \alpha \\
N \sin \alpha \\
0
\end{array}\right] \text { and }{ }_{I} \mathbf{F}_{\text {fric }}=\left[\begin{array}{c}
F_{\text {fric }} \sin \alpha \\
F_{\mathrm{imp}} \cos \alpha \\
0
\end{array}\right] .
\end{gathered}
$$

Finally, one writes Euler's equation with respect to the supporting point $O$

$$
\sum{ }_{B 3} \text { Force Moments }_{O}={ }_{B 3} \mathbf{I}_{O}\left(\frac{\mathrm{d}}{\mathrm{dt}} \boldsymbol{\omega}_{B 3}\right)+{ }_{B 3} \boldsymbol{\omega} \times\left({ }_{B 3} I_{O} \cdot{ }_{B 3} \boldsymbol{\omega}\right)
$$

and one solves the set of equations considering the angles $\Gamma$ and $\beta$ small. The motor has an independent control and is capable of delivering the necessary torque to keep the angular velocity $\dot{\theta}$ fixed. The differential equations are highly nonlinear, with many coupled terms, and the terms of the differential equations may be determined using the symbolic program Mapleß. However, we are more interested in the position of the center of shaft at the backup bearing plane and, using the transformation matrices, the coordinates are written as:

$$
\left[\begin{array}{c}
X \\
Y \\
Z
\end{array}\right]=\left({ }_{I} \mathbf{T}_{\Gamma}^{T}(t) \cdot{ }_{B 1} \mathbf{T}_{\beta}^{T}(t)-{ }_{I} \mathbf{T}_{\Gamma}^{T}(0) \cdot{ }_{B 1} T_{\beta}^{T}(0)\right) \cdot{ }_{B 2} \mathbf{r}_{O C}
$$

where the following initial conditions are taken into consideration: $t=0$, $\Gamma(0)=0$ and $\beta(0)=0$. The complete expressions for $\ddot{\Gamma}$ and $\ddot{\beta}$ are available in Appendix A.

\subsection{The backup bearing}

When the rotor drops and hits the rolling backup bearing, as seen in Figure 4, the friction force accelerates the inner race and the spheres.The angular position of the inner race, $\theta_{I r}$, is also a degree of freedom. Figure 4a illustrates a schematic view of balls and inner race and their velocities. 
Since the outer race is not moving and the spheres are not sliding on the touching point with it, point $O_{s p h}$, the tangential velocity is half of the one on the edge between the sphere and the inner race, point E. The force acting on one rolling bearing ball is shown in Figure $4 \mathrm{~b}$ and the following equations for one isolated ball are written in (16)-(18):

$$
\begin{aligned}
& \sum \text { Moment }_{O_{s p h}}=I_{s p h} \ddot{\theta}_{s p h} \\
& 2 r_{s p h} F_{i}=I_{s p h} \ddot{\theta}_{s p h}, \quad \text { since } v_{t}=\dot{\theta}_{i r} r_{2}=\dot{\theta}_{s p h} r_{s p h}, \\
& 2 r_{s p h} F_{i}=I_{s p h} \ddot{\theta}_{i r} \frac{r_{2}}{r_{s p h}}
\end{aligned}
$$

where $I_{s p h}=2 / 5\left(\pi r_{s p h}^{2}\right)+m_{s p h} r_{s p h}^{2}$. The Newton equation for the tangential direction leads to

$$
F_{i}+R_{o}=m_{s p h} a_{t}
$$

From (17) and (18),

$$
R_{o}+I_{s p h} \ddot{\theta}_{i r} \frac{r_{2}}{2\left(r_{s p h}\right)^{2}}=m_{s p h} \ddot{\theta}_{i r} r_{2} .
$$

Therefore, the angular acceleration of the inner race $\ddot{\theta}_{i r}$ can be obtained by taking into account the influence of all rolling spheres $j$ :

$$
\begin{aligned}
& \ddot{\theta}_{i r} I_{i r}=f r_{1}-r_{2} \sum_{j=1}^{N_{s p h}}\left(F_{i, j}\right), \\
& \ddot{\theta}_{i r} I_{i r}=f r_{1}-r_{2} N_{s p h}\left(\ddot{\theta}_{i r} \frac{I_{s p h} r_{2}}{2\left(r_{s p h}\right)^{2}}\right) \\
& \ddot{\theta}_{i r}\left(I_{i r}+N_{s p h} \frac{I_{s p h}}{2}\left(\frac{r_{2}}{r_{s p h}}\right)^{2}\right)=f r_{1} .
\end{aligned}
$$

\section{Simulation and nonlinear analysis}

\subsection{Integration in time}

The equations of motion (14), (21), (8), and (9) are integrated in time using a combined Matlab differential equation solver ode45 and a dedicated Runge-Kutta algorithm. The former is employed together with an Event function in order to find the exact moment of the impact. The latter is 
applied during the short period of impact. This is because it is a highly stiff problem and demands an enormous amount of time with very tight integration tolerances of the algorithm. Therefore, a test of convergence was performed and the adequate step time was set to $10^{-6} \mathrm{~s}$. During the impact, the relative velocity between the shaft and the inner race, $v_{\text {rel }}$, plays an important role. The friction force depends on it, and if the velocities match, there is no friction. Otherwise, the friction force is modeled as $F=$ $\mu N \operatorname{sign}\left(v_{\text {rel }}\right)$. The model parameters are shown in table 1 .

Table 1: Parameters set applied to the simulation.

\begin{tabular}{|l|l|}
\hline \multicolumn{2}{|c|}{ Parameter of the shaft } \\
\hline Mass without unbalance & $m=1.28 \mathrm{~kg}$ \\
Length to magnetic bearing & $r_{O C}=[0,0,0.384 \mathrm{~m}]$ \\
Length to rolling bearing & $r_{O B}=\left[r_{r} \cos (\alpha), r_{r} \sin (\alpha), 0.211 \mathrm{~m}\right]$ \\
Shaft diameter & $d_{r}=25 \mathrm{~mm}$ \\
\hline \hline \multicolumn{2}{|c|}{ Parameter of the rolling bearing } \\
\hline Inner diameter & $d_{1}=2 r_{1}=28 \mathrm{~mm}$ \\
Inner race outer diameter & $d_{2}=2 r_{2}=37 \mathrm{~mm}$ \\
Sphere radius & $r_{s p h}=5.0 \mathrm{~mm}$ \\
Impact stiffness & $k_{i m p}=2.5 \cdot 10^{10} \mathrm{~N} / \mathrm{m}^{3 / 2}$ \\
Friction coefficient & $\mu=0.20$ \\
Restitution coefficient & $e=0.90$ \\
\hline \hline \multicolumn{2}{|c|}{ Parameter of the inner and outer house } \\
\hline Mass from inner house & $m_{i h}=1.70 \mathrm{~kg}$ \\
Damping 1 & $c_{h}=7.04 \cdot 10^{2} \mathrm{Ns} / \mathrm{m}$ \\
\hline \hline Mass from outer housing & $M_{o h}=8.87 \mathrm{~kg}$ \\
Damping & $c_{v}=2.28 \cdot 10^{2} \mathrm{Ns} / \mathrm{m}$ \\
\hline \hline \multicolumn{2}{|c|}{ Force Sensor } \\
\hline Force transducer stiffness & $k_{f t}=8.3 \cdot 10^{7} \mathrm{~N} / \mathrm{M}$ \\
\hline
\end{tabular}

\subsection{Simulated results and experimental comparison.}

As mentioned before, in section 2.1 the test rig is a rotor suspended at one end by a removable passive bearing. From the moment that the magnetic force is removed, there are three distinct stages: a) the rotor free fall inside the bearing; b) the recurrence of contact between rotor and the inner race surface leading to the deformation of both of them and the appearance of an angular acceleration of the inner race; and finally c) the almost vanishing of the relative velocities between the inner race and shaft surfaces with the rotor finding a steady state condition at the bottom of the backup bearing. In 

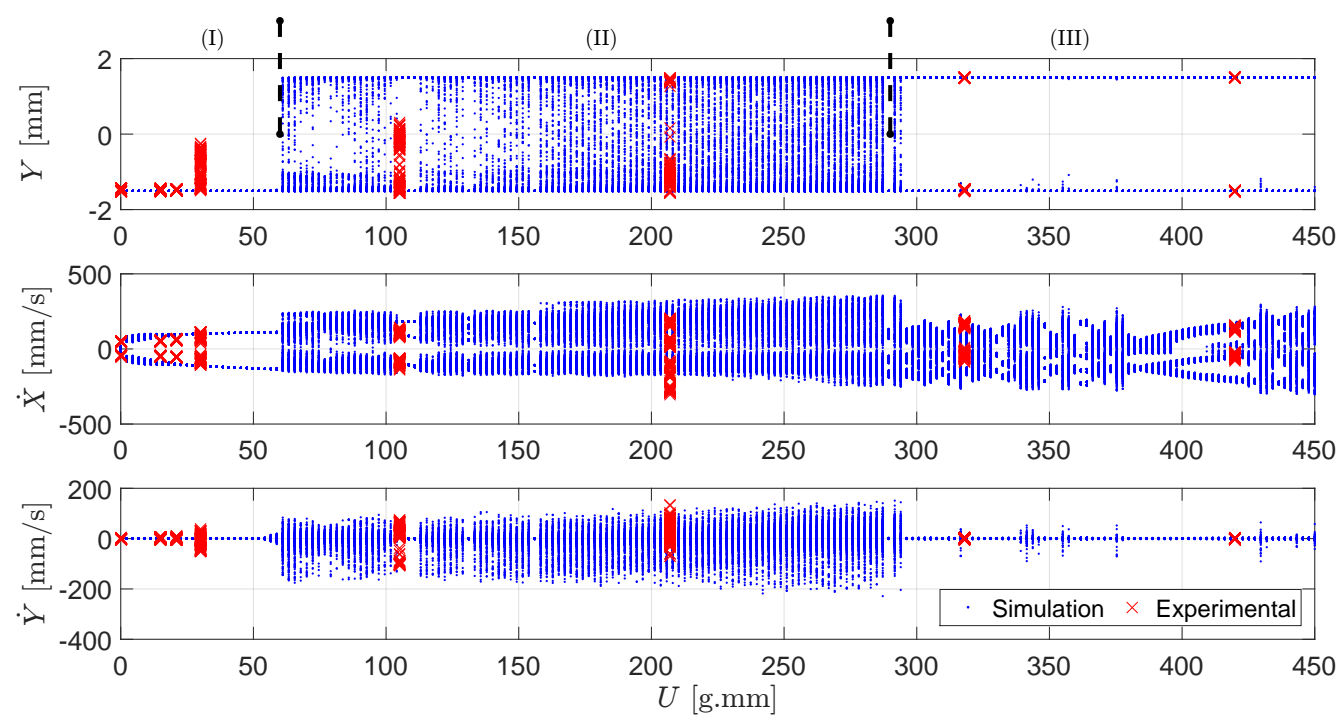

Figure 5: $10 \mathrm{~Hz}$ simulated bifurcation for different unbalance levels together with 8 different experimental tests with unbalance levels of: $U_{1}=0.0$ g.mm, $U_{2}=15$ g.mm, $U_{3}=21$ g.mm, $U_{4}=30$ g.mm, $U_{5}=105$ g.mm, $U_{6}=207$ g.mm, $U_{7}=318$ g.mm and $U_{8}=420$ g.mm.

fact, since the rotor keeps executing small translational movements around the equilibrium state, rarely will the tangential velocities of the shaft and the inner race coincide. This occurs in spite of the fact that the steady-state general dynamical behavior of the rotor changes significantly according to the unbalance level. After removing the magnetic force, the rotor falls down and impacts several times on the surface of the inner race. The energy will be dissipated by the damping from the coupling between the inner and outer housing and due to the impact with the compliant surface.

The changes in the steady-state behavior can be evaluated more explicitly with a Poincaré map, as seen in Figure 5. The variables $Y, \dot{X}$ and $\dot{Y}$ are sampled every time the center of the shaft crosses the vertical line; in other words, when $X=0$. The first plot on the top of Figure 5 shows the vertical position, $Y$, through which the rotor crosses the vertical line as a function of the control variable, i.e., the unbalance $U$.

Overall, the phenomena are captured by both experiment and simulation. We can divide the bifurcation diagram into three specific regions. First, the rotor center is always crossing at the bottom of the shaft at $Y=-1.5 \mathrm{~mm}$ in region I. Suddenly, there is a change and more crossings at the vertical line occur. This can be seen in region II of figure 5. The more unbalance, the more 
evident the fact that the center of the shaft performs chaotic trajectories. In the simulation it is expected that the rotor will cross from the bottom to the top of the bearing, but the mechanical set up shows a gradual increase in the vertical crossing as the unbalance gets bigger until the rotor is able to perform a full whirl. At the right end of the plot, region III, the rotor is only executing a full whirl so the rotor is crossing the vertical line in the extremes, $Y=-1.5$ to $Y=1.5 \mathrm{~mm}$. In the following plots, the horizontal and the vertical crossing velocity, $\dot{X}$ and $\dot{Y}$, are plotted against the unbalance and, once again, the experimental and the simulation measurement tend to agree with each other.

It can be seen that the unbalance plays a considerable role in the dynamics of the shaft and its size can strongly influence the dynamical pattern. The initial conditions were kept the same in experiments and simulations but in the experiments the rotor starts in contact with the bearing and the magnetic bearing is removed, while in the simulations, we start with the rotor at the center and the magnetic bearing is turned off later. From the start until the magnets are removed the orbit is painted in black, later, the color is red for one second of simulation, which then changes to blue.

The first case is presented in Figure 6, where there is no additional mass. The shaft is constantly in contact with the bearing inner race just oscillating. The numerical result shows the shaft falling and impacting the inner race and after some hits it stays on the bottom of the bearing. The difference between them exists because there is a misalignment at the coupling between the shaft and the motor.

Next, in Figure 7 we see a discrepancy between the experiment and the numerical result. While numerically it is shown that the rotor is whirling, falling and hitting a great number of times, the test rig developed a less complex orbit, in which the center of the shaft makes a quasi-periodic motion. One can see that the hits are concentrated around three regions. An additional increase in unbalance changes the orbit generated by the test rig and matches with the expected behaviour as demonstrated by the simulation in Figure 8. The chaotic behaviour shows some similarity when comparing numerical and experimental results.

Finally, Figures 9 and 10 reveal the last observed change in the rotor trajectories. The unbalance is high enough to maintain the rotor always in contact with the inner race, after the initial hits. Both the experiment and the numerical results are in clear accordance. Besides, there is a predominance of the forward whirl in both of them. The reason for the recurrence of the 
forward whirl is that the friction force is at a "stick" phase and therefore its magnitude is small and the wall is moving.

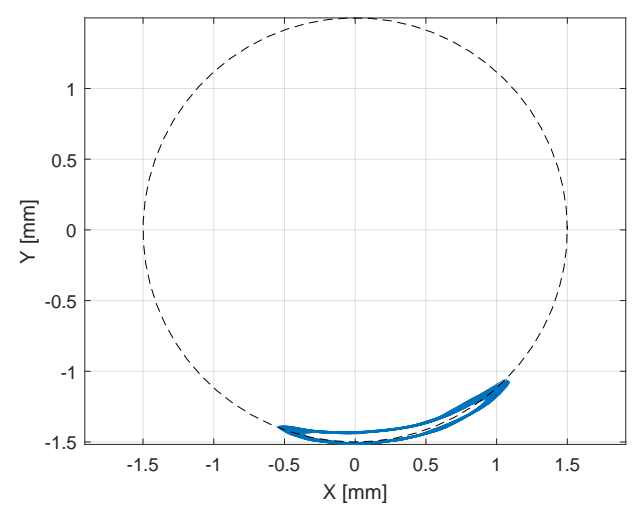

a)

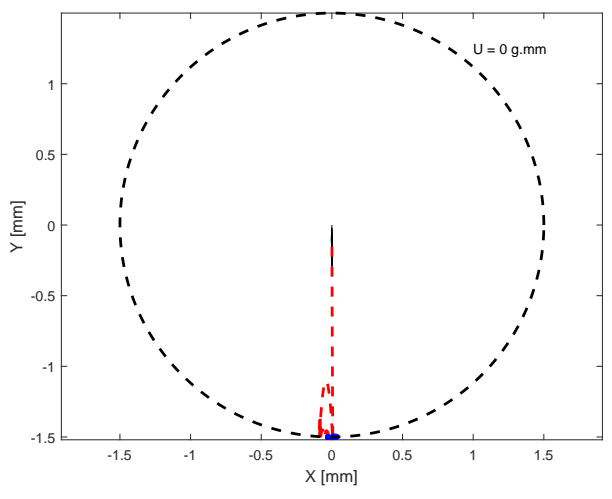

b)

Figure 6: Angular velocity $10 \mathrm{~Hz}$. Experimental a) and simulated b) orbit with no unbalance weight. ( $U=0.0$ g.mm).

To visualize the existence of chaotic trajectories performed by the rotor center, a double-sided spectrum is displayed in Figure 11. Each graph corresponds to one red unbalance level in Figure 5. At low levels the heights of the peaks are approximately the same. Several levels of unbalance are chosen; the cases $U=0.0$ g.mm, $U=15.0$ g.mm, $U=21.0$ g.mm, and $U=30.0$ g.mm represent region I. The noise clearly rises when the unbalance is $U=105.0$ g.mm and $U=207.0$ g.mm from region II, which is a clear indication that chaos is happening. In region III higher unbalance levels, $U=210.0$ g.mm and $U=420.0$ g.mm, show clearly higher peaks at $\Omega=-10.0 \mathrm{~Hz}$ than the opposite pair, confirming the predominance of a full whirl in the same direction as the rotor spinning. Negative values of $\omega$ mean the same spinning direction of the rotor.

As mentioned in section 2.2, the test rig has four force transducers positioned between the inner and outer housing. Their signals are converted to newtons and are sampled simultaneously with the other signals. For the same unbalance levels from figures 6 to 10, the horizontal (blue) and the vertical force (red) present on the bearing's inner race is plotted in Figure 12. Every addition in the unbalance increases the transient time until the steady state disappears for a level of unbalance belonging to region II of the bifurcation diagram. At a low level of unbalance there is a predominance of 


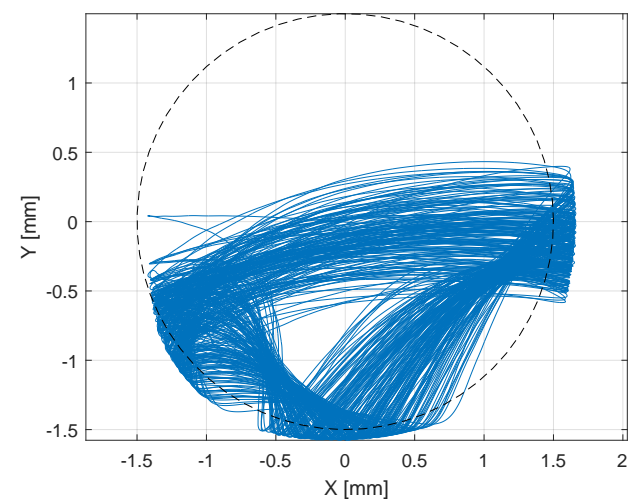

a)

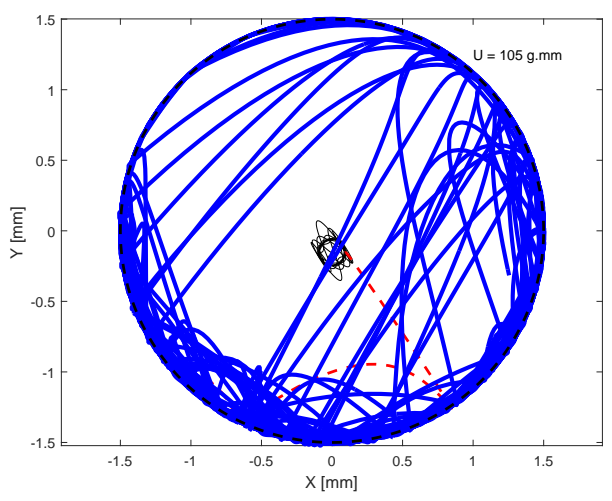

b)

Figure 7: Angular velocity $10 \mathrm{~Hz}$. Experimental a) and simulated b) orbit with unbalance magnitude of $U=105.0$ g.mm.

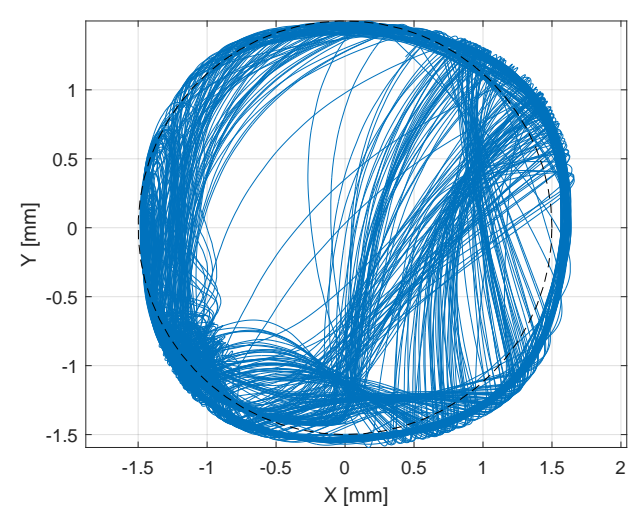

a)

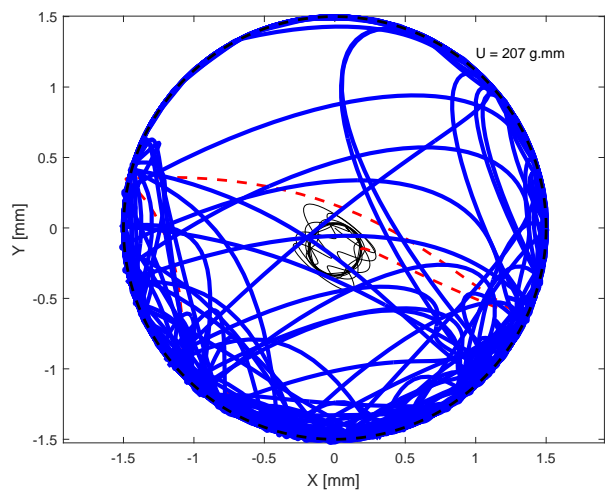

b)

Figure 8: Angular velocity $10 \mathrm{~Hz}$. Experimental a) and simulated b) orbit with unbalance magnitude of $U=207.0$ g.mm.

the vertical force, since the rotor is only moving on the bottom of the backup bearing.

Figure 12 demonstrates the effect of chaotic cases, in which the inner race receives multiple high magnitude impacts. The magnitude of the force reaches up to 200 newtons in each direction, which is considerably high for a rotor whose weight is only $12.74 \mathrm{~N}$. This situation changes for the regions III cases, since the shaft is always in contact with the surface of the inner race 


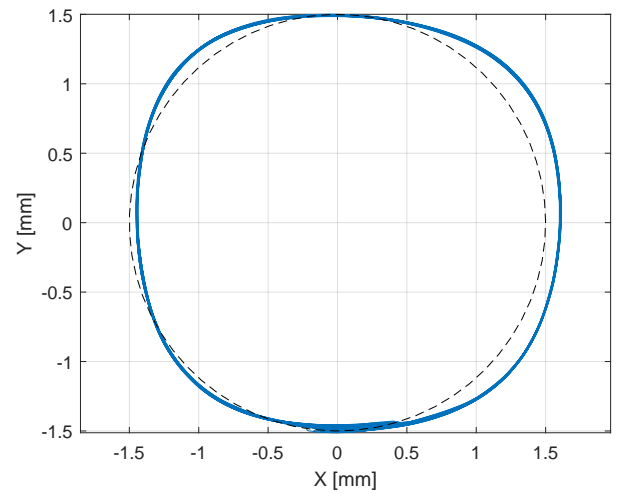

a)

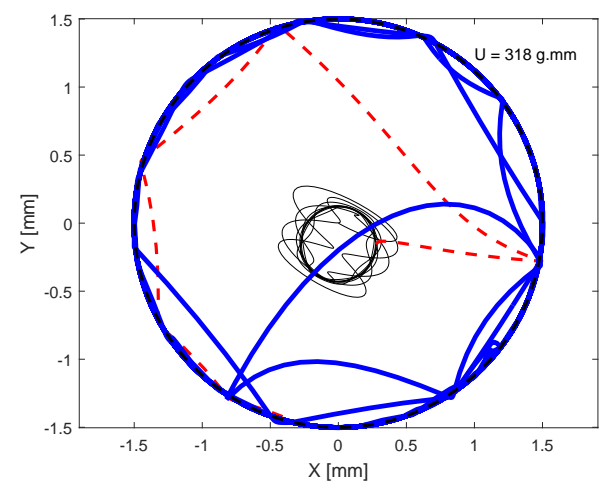

b)

Figure 9: Angular velocity $10 \mathrm{~Hz}$. Experimental a) and simulated b) orbit with unbalance magnitude of $U=318.0$ g.mm.

and is no longer impacting. The level of the forces oscillates between -20 to $20 \mathrm{~N}$ for both horizontal and vertical forces. The bearing has only to be able to withstand the centrifugal force in order not to break.

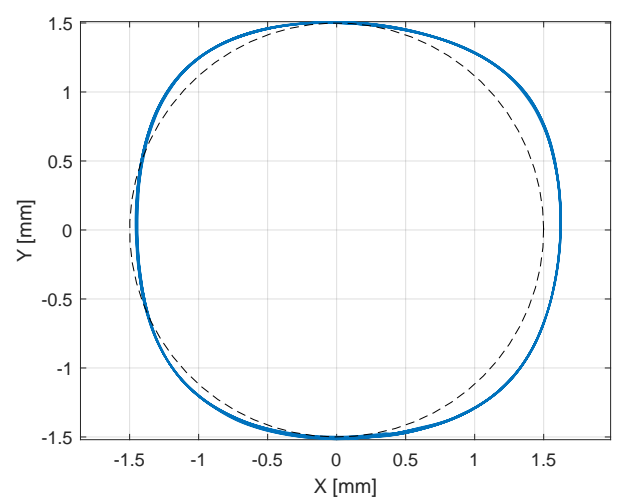

a)

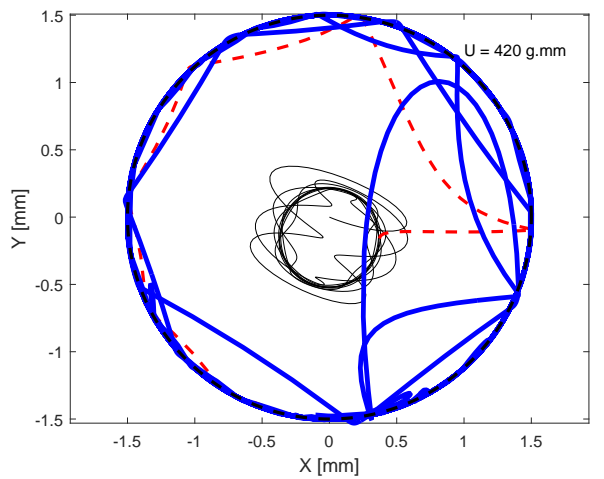

b)

Figure 10: Angular velocity $10 \mathrm{~Hz}$. Experimental a) and simulated b) orbit with unbalance magnitude of $U=420.0$ g.mm. 

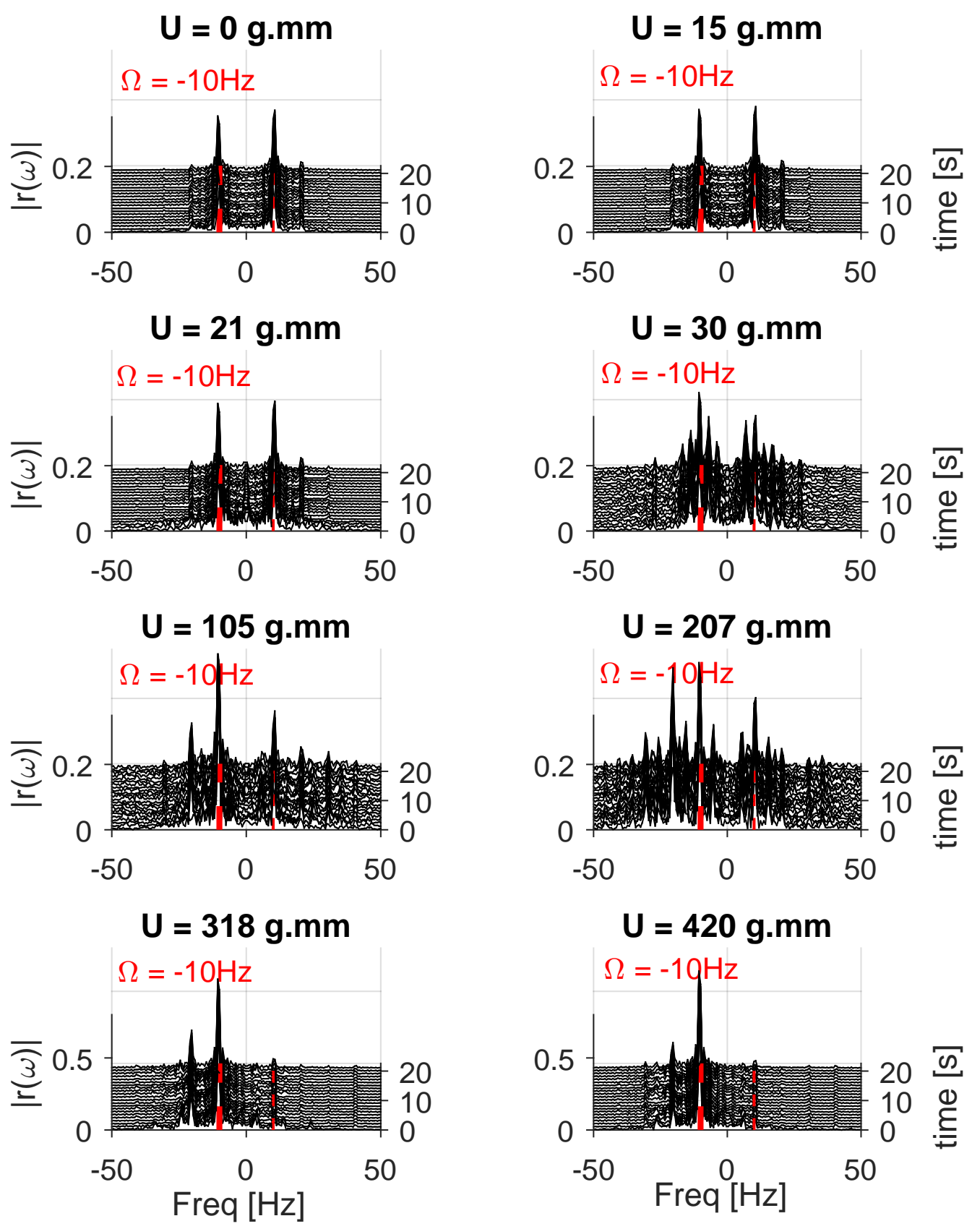

Figure 11: $10 \mathrm{~Hz}$ double sided spectrum. Effect of the unbalance in the general behavior of the rotor; from oscillatory movement, to chaos and full whirl. 

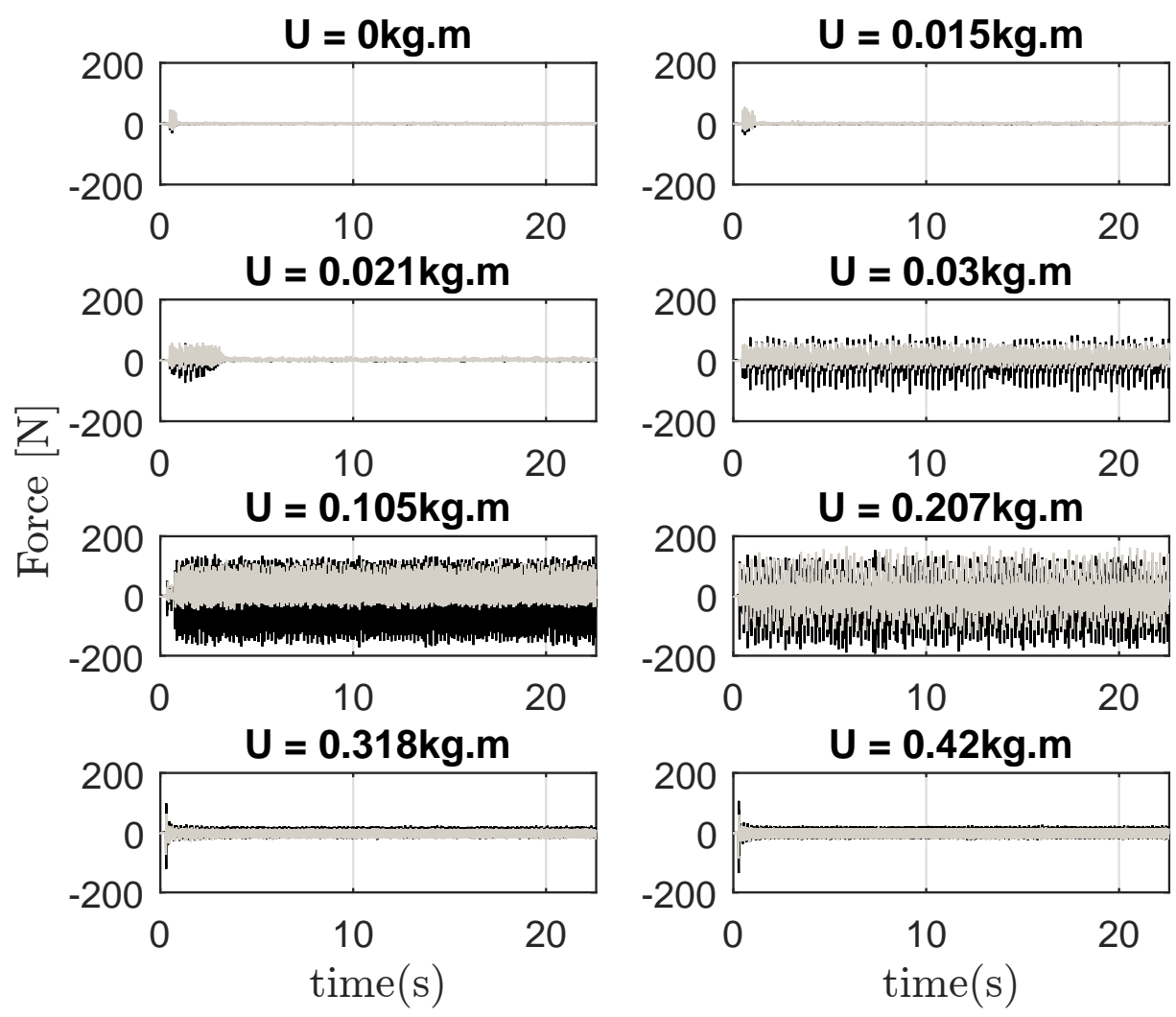

Figure 12: Force analysis for $10.0 \mathrm{~Hz}$ as angular velocity. Light gray is the resultant vertical force and black the horizontal force. The transient time takes longer to cease at every increase in the unbalance. Chaotic cases show high impact magnitude forces, but high unbalance lowered them, when the rotor is permanently in contact. 


\section{Conclusion}

From the theoretical and experimental nonlinear analysis carried out in this paper, one can conclude that the rotor-backup bearing system has three distinguished dynamical behaviors depending on the level of rotor unbalance. For region I, characterized by low unbalance level from $(0,60]$ g.mm, simple oscillatory movements of the rotor center at the bottom of the backup bearing are seen. For region II with unbalance levels from (60 to 280] g.mm, chaotic motions are followed by impacts between the rotor and backup inner race surface. Finally, for region III characterized by high unbalance levels, full forward whirl of the rotor center takes place.

The magnitude of the forces at the three different regions changes and supports the idea that it may damage the backup bearing. At low levels of unbalance, the magnitude of the forces varies slightly in time and just enough to support the fallen rotor. Chaotic cases cause multiple impacts, which means that the contact forces are always high with a short duration. Although a rotor with big unbalance remains in contact with the inner race, the strength of the force drops significantly.

\section{Acknowledgement}

The authors express their acknowledgement to CNPq through the Science Without Borders Program with the process number: 249728/2013-3, which sponsored the elaboration of this paper. 


\section{Appendix A. Equations for $\ddot{\Gamma}$ and $\ddot{\beta}$}

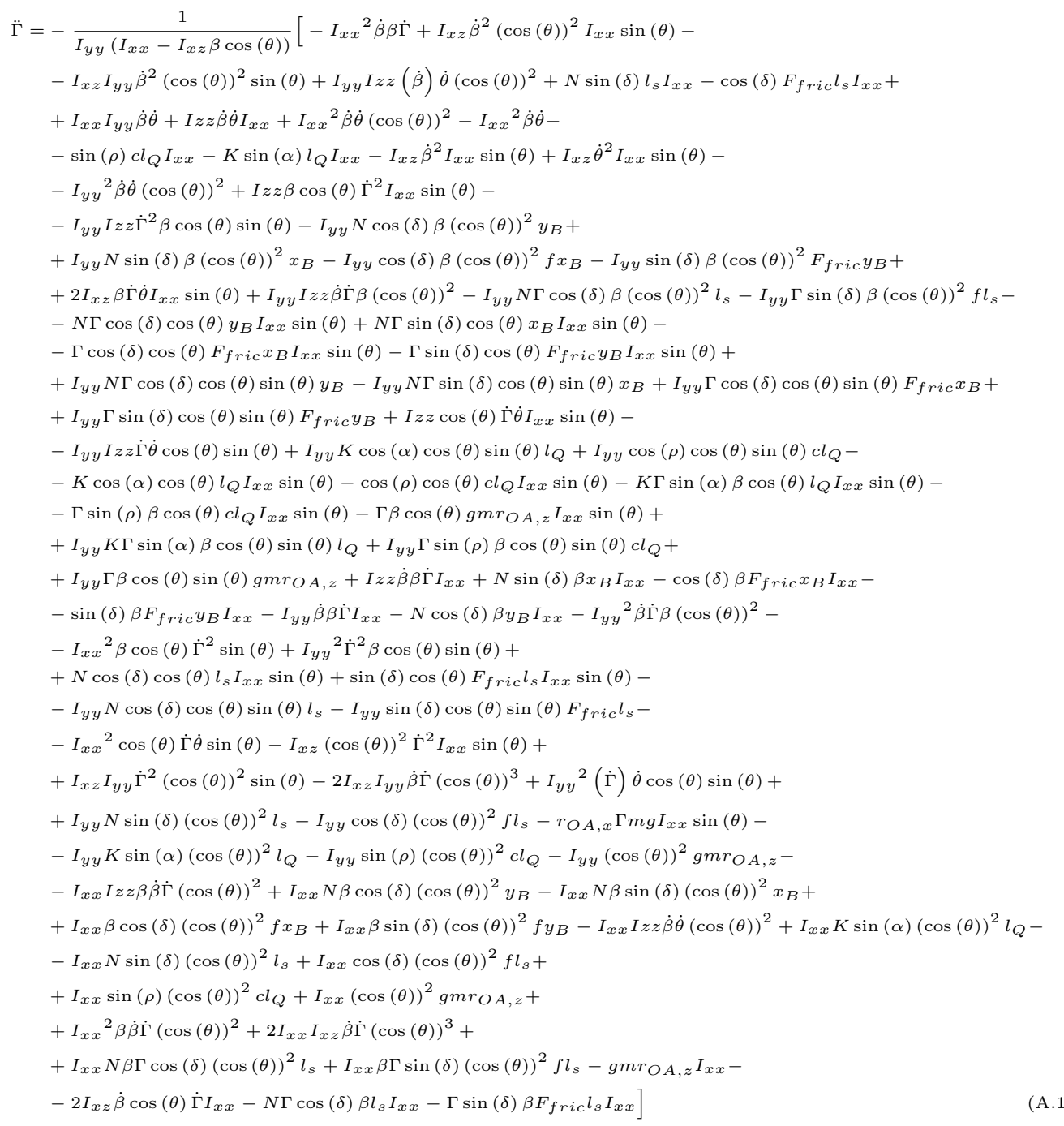




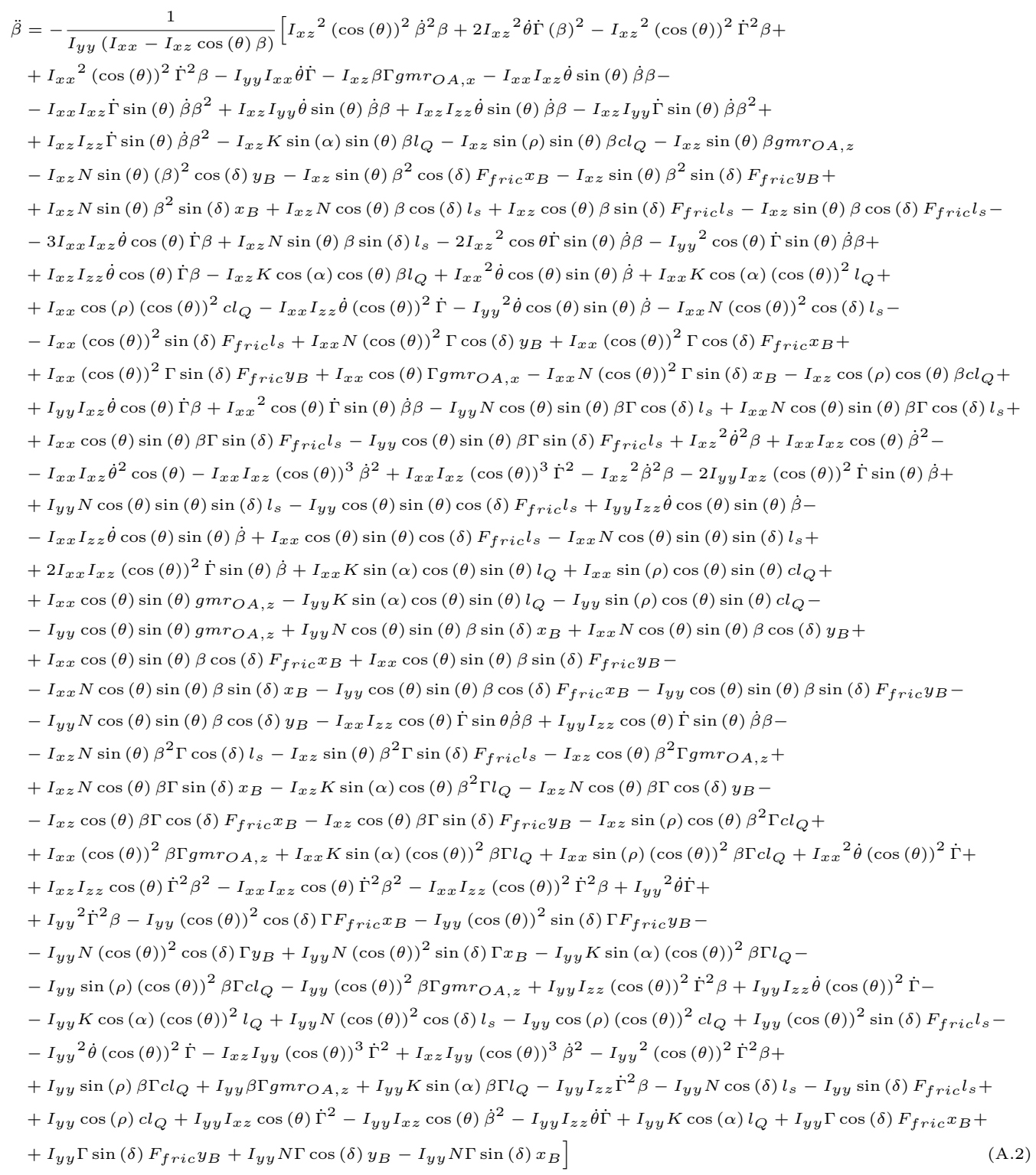

\section{References}

[1] Black, H. F., 1968. Interaction of a whirling rotor with a vibrating stator across a clearance annulus. Arch. J. Mech. Eng. Sci. 1959-1982 (vols 1- 
23) $10(1), 1-12$.

[2] Choy, F. K., Padovan, J., 1987. Non-linear transient analysis of rotorcasing rub events. Journal of Sound and Vibration 113, 529-545.

[3] Cole, M. T., Keogh, P. S., Burrows, C. R., 2001. The dynamic behavior of a rolling element auxiliary bearing following rotor impact. ASME. J. Tribol. 2 (124), 406-413.

[4] Fonseca, C. A., Weber, H., Fleischer, P., Santos, I., 2015. Analyzing the use of pins in safety bearings. J. Braz. Soc. Mech. Sci. Eng. 37, $1425-1434$.

[5] Fumagalli, M. A., 1997. Modelling and measurement analysis of the contact interaction between a high speed rotor and its stator. Ph.D. thesis, ETH - Swiss Institute Of Technology Zurich.

[6] Gasch, R., Nordmann, R., Pfützner, H., 1975. Die harte Statorberührung - Fanglager [The strong stator-contact - Catcher Bearing.]. Springer Berlin Heidelberg, Berlin, Heidelberg, pp. 555-573.

[7] Gasch, R., Nordmann, R., Pfützner, H., 2002. Rotordynamik, 2nd Edition. Springer Verlag, Berlin.

[8] Ginzinger, L., Ulbrich, H., 2007. Control of a rubbing rotor using an active auxiliary bearing. Journal of Mechanical Science and Technology 21 (6), 851-854.

[9] Goldman, P., Muszynska, A., 1994. Chaotic Behavior of Rotor/Stator Systems With Rubs. J. Eng. Gas Turbines Power 116 (3), 692.

[10] Hawkins, L., McMullen, P., Vuong, V., May 2007. Development and testing of the backup bearing system for an AMB energy storage flywheel. In: ASME (Ed.), Proceedings of GT2007.

[11] Inayat-Hussain, J. I., 2010. Nonlinear dynamics of a magnetically supported rigid rotor in auxiliary bearings. Mechanism and Machine Theory 45 (11), 1651-1667.

[12] Ishii, T., Kirk, R. G., 1996. Transient Response Technique Applied to Active Magnetic Bearing Machinery During Rotor Drop. Transactions of the ASME 118 (April 1996). 
[13] Jacquet-Richardet, G., Torkhani, M., Cartraud, P., Thouverez, F., Nouri-Baranger, T., et al.., 2013. Rotor to stator contacts in turbomachines. review and application. Mechanical Systems and Signal Processing 40 (2), 401-420.

[14] Jiang, J., Ulbrich, H., 2005. The Physical Reason and the Analytical Condition for the Onset of Dry Whip in Rotor-to-Stator Contact Systems. J. Vib. Acoust. 127 (6), 594.

[15] Johnson, D. C., 1962. Synchronous whirl of a vertical shaft having clearance in one bearing. Arch. J. Mech. Eng. Sci. 1959-1982 (vols 1-23) 4 (1), 85-93.

[16] Keogh, P. S., may 2012. Contact dynamic phenomena in rotating machines: Active/passive considerations. Mech. Syst. Signal Process. 29, $19-33$.

[17] Lahriri, S., Santos, I. F., 2012. Experimental quantification of dynamic forces and shaft motion in two different types of backup bearings under several contact conditions. Journal Mechanical Systems and Signal Processing.

[18] Lankarani, H. M., Nikravesh, P. E., 1990. A Contact Force Model With Hysteresis Damping for Impact Analysis of Multibody Systems. J. Mech. Des. $112(3), 369$.

[19] Muszynska, A., 1989. Rotor-to-stationary element rub-related vibration phenomena in rotating machinery - literature survey. Shock and Vibration Digest 21 (3), 3-11.

[20] Piccoli, H. C., Weber, H. I., 1998. Experimental observation of chaotic motion in a rotor with rubbing. Nonlinear Dyn. 16 (1), 55-70.

[21] Pradetto, J. C., Schmied, J., 1992. Behaviour of a one ton rotor being dropped into auxiliary bearings. In: Proceeding 3rd Int. Symp. Magn. Bear. pp. 145-156.

[22] Schweitzer, G., March 2009. Applications and research topics for active magnetic bearings. Proc. IUTAM-Symp. on Emerging Trends in Rotor Dynamics. 
[23] Schweitzer, G., Maslen, E. H., 2009. Magnetic Bearings, 1st Edition. Springer-Verlag Berlin Heidelberg.

[24] Siegl, G., Tzianetopoulou, T., Denk, J., 2016. Simulation and experimental validation of a 9 ton AMB rotor landing in rolling element backup bearings. Mechanical Engineering Journal 3 (1), 15-00136-15-00136.

[25] Simon, U., 2001. Rotor stator kontakt in polygonförmigen fanglagern [rotor - stator - contact in polygonal catcher bearings]. Ph.D. thesis, Technischen Universität CaroloWilhelmina zu Braunschweig.

[26] Sun, G., Palazzolo, A., Provenza, A., Montague, G., 2004. Detailed ball bearing model for magnetic suspension auxiliary service. Journal of Sound and Vibration 269 (35), 933 - 963.

[27] van Rensburg, J. J. J., 2014. Delevitation modelling of an active magnetic bearing supported rotor. Phd thesis, North-West University.

[28] Wang, X. X., Noah, S. S., 1998. Nonlinear dynamics of a magnetically supported rotor on safety auxiliary bearings. ASME. J. Vib. Acoust. 2 (120), 596-606.

[29] Wojciech, M. S., 1986. Dynamisches Verhalten eines schnell drehenden Rotors bei Anstreifvorgangen. Ph.D. thesis, TU Karlsruhe.

[30] Zülow, D., Liebich, R., 2009. Ein aussenrollenlager als fanglagerkonzept für magnetgelagerte rotoren [an external ball bearing as catcher bearing concept]. In: SIRM 8. Internationale Tagung Schwingungen in rotierenden Maschinen, Wien, Austria, paper-ID 11. 